\title{
Review of: "Growth in infants, children and adolescents with unilateral and bilateral cerebral palsy"
}

Tomoki Maeda ${ }^{1}$

1 Oita University

Potential competing interests: The author(s) declared that no potential competing interests exist.

The authors investigated the longitudinal growth in children with CP. The results are clear and interesting. There are limitations of retrospective study design, but there is a little information to derive insights from the results.

As the authors mentioned, it is likely that the insufficient growth spurt with puberty is associated with depressed growth in term born CP cases. However, there is no information on secondary sexual characteristics. For example, gender differences, history of precocious puberty, menarche age, etc. I think it would be better to analyze those data as well.

Regarding the type of $\mathrm{CP}$, I suggest to investigate the growth difference not only in bilateral CP or unilateral CP but also in the type (spastic, diakinetic and hypotonic) of CP that reflects the etiology of injury. I think this investigation would provide useful information for considering the factors that contribute to the growth characteristics of CP. 